\title{
Comparaison de cinq méthodes pour extraire les lipides du lactosérum et de ses dérivés
}

\author{
C Théodet, G Gandemer* \\ INRA, laboratoire d'étude des interactions des molécules alimentaires, \\ BP 527, 44026 Nantes Cedex 03, France
}

(Reçu le 28 mai 1990; accepté le 1er octobre 1990)

\begin{abstract}
Résumé- Les résultats de 5 méthodes d'extraction des lipides appliquées au lactosérum et à un concentré lipoprotéique de lactosérum sont comparés. Les paramètres pris en compte sont la teneur en lipides, les proportions relatives de lipides neutres et de phospholipides, la composition en acides gras de ces 3 fractions et la répartition en classes des phospholipides.

La méthode de Clark et al (1982) est la mieux adaptée à l'analyse quantitative et qualitative des lipides du lactosérum et de ces co-produits, mais cette méthode est fastidieuse. Pour les analyses de routine, les méthodes de Röse-Gottlieb et de Wolff et Castera-Rossignol (1987) peuvent être utilisées. Toutefois, elles sous-estiment le taux d'acides gras polyinsaturés des phospholipides. De plus, la méthode de Wolff et Castera-Rossignol (1987) surévalue nettement la teneur en lipides des 2 produits. La méthode de SBR est à proscrire parce qu'elle altère très fortement les phospholipides. La méthode de Folch et al (1957) n'est pas adaptée à l'extractior des lipides des co-produits riches en lipides.
\end{abstract}

lipide / phospholipide / extraction / lactosérum / concentré lipoprotéique

Summary - Comparison of 5 methods for lipid extraction from whey and its byproducts. Results of 5 methods for lipid extraction from whey and a high lipid content by-product were compared. The following parameters were taken into account: lipid content, relative proportions of neutral lipids and phospholipids, fatty acid composition of these fractions and phospholipid composition. The method of Clark et al (1982) is the most suitable method for quantitative and qualitative analysis of lipids from whey and its by-products, but it is time-consuming. For routine analysis, the methods of Röse Gottlieb and Wolff and Castera-Rossignol (1987) should be used. However, polyunsaturated fatty acid content of phospholipids and underestimated. Moreover, lipid content of both the products are overestimated when Wolff are Castera's method is used. Use of the SBR method should be avoided as a large amount of phospholipids are destroyed during the procedure. The method of Folch et al (1957) is not suitable for lipid extraction from the whey by-product with a high lipid content.

lipid / phospholipid / extraction / whey / byproduct

\footnotetext{
* Correspondance et tirés à part
} 


\section{INTRODUCTION}

Les lipides du lactosérum et des ses dérivés sont très mal connus. En effet, les seules données disponibles se limitent à leur teneur en lipides (Cerbulis et al, 1972; Fauquant et al, 1985b). Or, ces lipides doivent être éliminés pour permettre le fractionnement ultérieur des protéines du lactosérum (Maubois et al, 1987). Même si des procédés ont été développés pour les éliminer (Fauquant et al, 1985a, 1985b), une meilleure connaissance de ces lipides est indispensable à l'optimisation des procédés de fractionnement des protéines du lactosérum et à la valorisation des fractions telles que les concentrés lipoprotéiques.

La caractérisation des lipides d'un aliment impose leur extraction. De cette étape initiale dépend la qualité des résultats de toutes les analyses pratiquées ultérieurement pour caractériser et quantifier les lipides (Zhukov et Vereshchagin, 1981). C'est pourquoi il faut disposer d'une méthode qui permette leur extraction totale sans les altérer. Le problème est rendu délicat par la faible teneur en lipides du lactosérum et l'existence de complexes lipides-protéines stables dans les concentrés lipoprotéiques.

Les méthodes les plus largement usitées pour l'extraction des lipides des produits laitiers sont basées sur les méthodes de Schmidt Bonzynski Ratzlaff (SBR) et de Röse-Gottlieb. Elles font appel à une hydrolyse acide ou basique du produit associée à un traitement thermique pour dissocier les complexes lipides-protéines. Or, la chaleur et les acides sont des facteurs susceptibles d'oxyder et d'hydrolyser les lipides, en particulier les phospholipides. C'est pourquoi les biologistes leur préfèrent des méthodes à froid reposant sur I'utilisation d'un mélange binaire de solvants comme le chloroforme-méthanol, le dichlorométhane-méthanol ou l'hexaneisopropanol (Folch et al, 1957; Hara et Radin, 1978; Marmer et Maxwell, 1981). Dans ces mélanges solvants, les alcools provoquent une dissociation des interactions lipides-protéines. Des modifications de ces méthodes ont été proposées par plusieurs auteurs pour les adapter aux produits laitiers (Timmen et Dimick, 1972; Clark et al, 1982; Maxwell et al, 1986; Wolff et Castera-Rossignol, 1987). Cependant à notre connaissance, elles n'ont jamais été testées pour l'extraction des lipides du lactosérum et de ses dérivés.

Ce travail a pour objet d'évaluer les performances de 5 méthodes pour extraire quantitativement sans les altérer les lipides du lactosérum et d'un concentré lipoprotéique. Les paramètres pris en compte sont : la teneur en lipides des substrats, les proportions relatives des lipides neutres et polaires de l'extrait lipidique ainsi que la composition en acides gras de ces fractions et la répartition en classes des phospholipides.

\section{MATÉRIEL ET MÉTHODES}

\section{Origine des matières premières}

Vingt cinq litres de lactosérum et de concentré lipoprotéique ont été fournis par la Coopérative laitière de la Région nantaise (Colarena, Herbignac).

Le lactosérum est issu d'une fabrication de fromage d'emmental. Le concentré lipoprotéique est la fraction de lactosérum obtenue par précipitation thermocalcique des "phospholipoprotéines" selon le procédé développé par Fauquant et al (1985a; 1985b). Cette technique est basée sur la propriété d'agrégation des phospholipoprotéines sous l'effet de la chaleur en présence de calcium $\left(50^{\circ} \mathrm{C}\right.$ pendant $8 \mathrm{~min}$, $\mathrm{CaCl}_{2} 1,2 \mathrm{~g} / \mathrm{kg}, \mathrm{pH} 7,3$ ); une microfiltration tangentielle permet la séparation de la fraction précipitée (concentré lipoprotéique) de la fraction 
soluble (microfiltrat). Le concentré lipoprotéique n'a été ni déminéralisé ni délactosé. Pour permettre leur conservation les 2 fractions ont été additionnées d'azide de sodium $(0,02 \%)$ et lyophilisées dans un appareil de laboratoire Virtis. Les échantillons sont conservés dans des flacons opaques à $4{ }^{\circ} \mathrm{C}$. La composition chimique de ces 2 fractions est donnée dans le tableau I.

Tableau I. Composition chimique du lactosérum et du concentré lipoprotéique (en g/100 g de matière sèche).

Chemical composition of the whey and high lipid content by-product (in g/100 g dry weight).

Lactosérum Concentré lipoprotéique

$\begin{array}{lrr}\text { Lipides } & 0,7 & 2,9 \\ \text { Protéines } & 16,4 & 21,8 \\ \text { Glucides } & 75,0 & 44,0 \\ \text { Cendres } & 8,2 & 22,4\end{array}$

\section{Préparation des échantillons}

La réhydratation est un préalable nécessaire à l'extraction quantitative des lipides dans les produits déshydratés (Christie, 1987). En effet, l'extraction directe des lipides sur la matière sèche conduit à une estimation par défaut de la teneur en lipides du produit (Castera et al, 1989). La quantité d'eau ajoutée varie beaucoup suivant les auteurs en fonction du produit et de la méthode d'extraction considérés. Toutefois, il n'est pas nécessaire de procéder à une réhydratation complète de l'échantillon. Des essais successifs nous ont permis d'établir que l'ajout de $20 \mathrm{ml}$ d'eau à $10 \mathrm{~g}$ de lyophilisat était satisfaisant pour la majorité des méthodes testées. La réhydratation est assurée en agitant le mélange pendant environ $2 \mathrm{~h}$, à l'aide d'un agitateur magnétique.

Dans le cas de la méthode de Röse-Gottlieb qui est la méthode normalisée pour le dosage des lipides du lactosérum (NF 04-346), nous nous en sommes tenus à la procédure de réhydratation décrite dans cette norme soit $1,5 \mathrm{~g}$ de lyophilisat pour $10 \mathrm{ml}$ d'eau.

Dans le cas de la méthode de Folch et al (1957), l'agitation de la suspension de l'échan- tillon hydraté dans le mélange chloroformeméthanol a donné naissance à une suspension stable qu'il est pratiquement impossible de filtrer confirmant les observations de Maxwell et al (1986). C'est pourquoi l'extraction a été effectuée directement sur le lyophilisat.

\section{Méthodes d'extraction}

Les méthodes que nous avons comparées se répartissent en 2 groupes :

- les méthodes de référence normalisées pour l'extraction des lipides dans les produits laitiers (SBR, Röse-Gottlieb);

- les méthodes à froid utilisées pour extraire les lipides dans les tissus biologiques ou les produits alimentaires avec des solvants binaires (Folch et al, 1957; Clark et al, 1982; Wolff et Castera-Rossignol, 1987).

\section{Méthodes de référence pour les produits laitiers}

La particularité de ces 2 méthodes consiste en une hydrol'ne à chaud des échantillons avant de procéder à l'extraction des lipides.

Dans la méthode de SBR (Norme AFNOR NF V 04-215), l'hydrolyse est réalisée en milieu chlorhydrique $(\mathrm{HCl} 11,5 \mathrm{~N})$ à $100^{\circ} \mathrm{C}$ pendant 30 min.

Dans la méthode de Röse-Gottlieb (Norme AFNOR, NF V 04-346), l'hydrolyse est effectuée en présence d'ammoniaque (25\%) pendant 15 min à $60-70^{\circ} \mathrm{C}$.

Après ce traitement, les lipides sont extraits par un mélange d'oxyde diéthylique et d'éther de pétrole $(1: 1, v / V)$ après ajout d'éthanol à l'hydrolysat. Pour les 2 méthodes, il n'est procédé à aucune purification ultérieure de l'extrait lipidique pour éliminer les contaminants non lipidiques.

\section{Méthodes à froid}

Ces méthodes font appel à un mélange binaire composé d'un bon solvant des lipides (chloroforme, hexane, etc) et d'un solvant plus polaire qui permet de rompre les liaisons lipides- 
protéines (méthanol, isopropanol, etc). L'extraction est réalisée à froid. L'extrait lipidique est toujours purifié.

Dans la méthode de Folch et al (1957), le mélange solvant est composé de chloroforme/ méthanol $(2: 1, v / v)$. L'extrait est purifié par lavage de la phase organique avec une solution saline ( $\mathrm{NaCl}, 0,73 \%)$.

Dans la méthode de Wolff et CasteraRossignol (1987), les lipides sont extraits avec le mélange solvant hexane/isopropanol $(3: 2, \mathrm{v} /$ v). L'élimination des contaminants est réalisée par filtration de l'extrait lipidique sur une colonne sèche (célite $545 / \mathrm{Na}_{2} \mathrm{SO}_{4}$ ), 5/20). Cette technique est rapide $(2 \mathrm{~h})$.

La méthode de Clark et al (1982) est inspirée directement de la méthode de Folch et al (1957). Elle utilise les mêmes solvants et une procédure de purification de l'extrait lipidique similaire. Cependant, toutes les étapes de l'extraction se déroulent en ampoule à décanter. En effet, l'échantillon est mis en suspension dans le solvant à l'intérieur de l'ampoule évitant la phase d'homogénéisation mécanique responsable de la formation des suspensions stables rencontrées dans la méthode de Folch et al (1957). L'extraction est réalisée par épuisement de l'échantillon lors de 2 phases successives de décantation et de remise en suspension dans du solvant frais. L'inconvénient de cette technique est la durée des décantations (2-3j).

Dans le procédé de clarification du lactosérum, Fauquant et al (1985a; 1985b) provoquent une agrégation des phospholipoprotéines en présence des ions calcium sous l'action d'un traitement thermique. C'est pourquoi nous avons pensé qu'une complexation des ions $\mathrm{Ca}^{2+}$ par I'EDTA ou leur solubilisation en milieu acide, seraient de nature à favoriser l'extraction des lipides. Au cours de la phase de réhydratation, les échantillons ont été additionnés d'EDTA (200 mmol. $\mathrm{I}^{-1}$ ), ou d'acide chlorhydrique pour ajuster leur $\mathrm{pH}$ à 4,6. Environ $1 \mathrm{~h}$ après ces opérations, les lipides sont extraits suivant la méthode de Clark et al (1982).

Toutes les extractions ont été réalisées au cours d'une même quinzaine pour éviter qu'une oxydation toujours possible des lipides des échantillons n'introduise un biais dans la comparaison des méthodes. Pour chaque méthode, 4 extractions ont été effectuées à partir de 5-10 $g$ de lyophilisat de lactosérum et de concentré lipoprotéique. Les solvants sont éliminés à l'aide d'un évaporateur rotatif, puis sous un léger courant d'azote. La teneur en lipides est déterminée par pesée et exprimée en $\mathrm{g} / 100 \mathrm{~g}$ de matière sèche.

\section{Fractionnement en lipides neutres et polaires}

L'extrait lipidique est fractionné en lipides neutres et polaires sur cartouche de silice (Seppack, Waters SA) selon la méthode de Juaneda et Rocquelin (1985). Les lipides neutres sont élués par du chloroforme, puis sont quantifiés par pesée. Les phospholipides sont élués par le méthanol; ils sont quantifiés par dosage du phosphore directement dans l'extrait lipidique total selon Bartlett (1959). La teneur en phospholipides est calculée en multipliant le taux de phosphore lipidique par le coefficient 25.

Les contenus en lipides neutres et phospholipides sont exprimés en $\mathrm{g} / 100 \mathrm{~g}$ de matière sèche.

\section{Répartition en classes de phospholipides}

La répartition en classes de phospholipides est déterminée par chromatographie liquide haute pression. L'appareil comprend 2 pompes et une vanne d'injection rhéodyne équipée d'une boucle de $20 \mu \mathrm{l}$. La colonne en acier de $25 \mathrm{~cm}$ de long et de $4,5 \mathrm{~mm}$ de diamètre est remplie de silice (Lichrospher, $5 \mu \mathrm{m}$ ). Les phospholipides sont détectés à l'aide d'un détecteur ả diffusion de lumière (DDL 10, Cunow) relié à un système d'intégration Nelson. L'élution des phospholipides est obtenue en $25 \mathrm{~min}$. Les phases mobiles A et B sont composées respectivement de chloroforme pur et du mélange chloroforme/méthanol/eau/ammoniaque (92:5:2:1, v/ $\mathrm{v} / \mathrm{v} / \mathrm{v})$, la proportion de solvant B passe de $0 \%$ à $20 \%$ en 10 min puis de $20 \%$ à $100 \%$ en $10 \mathrm{~min}$. Le débit de la phase mobile est de $1,5 \mathrm{ml} / \mathrm{min}$. La quantification des phospholipides par diffusion de lumière nécessite un étalonnage de l'appareil. Cette opération a été décrite antérieurement (Leseigneur et al, 1989). Les conditions optimales de fonctionnement du détecteur pour la quantification des phospholipides sont les suivantes : pression du gaz de nébulisation, 2,2 bar; pression du gaz auxiliaire, 0,5 bar; tempéra- 
ture de nébulisation du solvant $40^{\circ} \mathrm{C}$. Dans ces conditions opératoires, la réponse du détecteur est une fonction linéaire de la concentration dans une gamme comprise entre 5 et $200 \mu \mathrm{g}$ de phospholipides par pic. Les résultats sont exprimés en \% de la surface des phospholipides totaux.

\section{Composition en acides gras des différentes fractions}

La composition en acides gras des fractions est déterminée par chromatographie en phase gazeuse de leurs esters méthyliques. Ceux-ci sont préparés par transestérification dans le méthanol / $\mathrm{BF}_{3}$ à $14 \%$ suivant la technique de Morrison et Smith (1964). L'analyse est réalisée à l'aide d'un chromatographe DANI 6500 (StangInstruments, France) équipé d'un injecteur PTV, d'un détecteur à ionisation de flamme. II est connecté à un intégrateur ENICA 21 (Delsi Instrument, France). Une colonne capillaire en silice fondue de $30 \mathrm{~m}$ de longueur et $0,32 \mathrm{~mm}$ de diamètre interne a été utilisée (Superox II, Alltech). L'épaisseur du film de la phase stationnaire polaire (Polyéthylène glycol) est de 0,25 $\mu \mathrm{m}$. L'injection est réalisée en mode division (1/ 50). La température du four est maintenue 4 min à $150^{\circ} \mathrm{C}$, puis programmée à $5{ }^{\circ} \mathrm{C} /$ min jusqu'à $180{ }^{\circ} \mathrm{C}$ où elle est stabilisée jusqu'à la fin de l'analyse. Les températures de l'injecteur et du détecteur sont de $250^{\circ} \mathrm{C}$. La pression du gaz vecteur en tête de colonne (hydrogène) est de 0,6 bar.

Les esters méthyliques d'acides gras ont été identifiés par comparaison de leur temps de rétention avec ceux d'esters méthyliques de solutions étalons.

Seuls les acides gras ayant au moins 10 atomes de carbone ont été pris en compte, les conditions chromatographiques ne permettant pas la séparation des acides gras à chaîne courte du front de solvant. La composition en acides gras est exprimée en pour cent des surfaces des esters méthyliques injectés.

\section{Analyses statistiques}

La comparaison des résultats obtenus suivant les différentes méthodes est réalisée par ana- lyse de variance à 1 facteur (méthode) à 5 niveaux. Les calculs ont été effectués à l'aide du logiciel commercialisé par l'ITCF (STAT-ITCF).

\section{RÉSULTATS}

\section{Teneur en lipides (tableau II)}

Le lactosérum contient $0,7-0,8 \mathrm{~g}$ de lipides pour $100 \mathrm{~g}$ de matière sèche alors que le concentré lipoprotéique en est $3-4$ fois plus riche $(2,5-3,0 \mathrm{~g} / 100 \mathrm{~g}$ de matière sèche). Pour les 2 produits, l'analyse de variance révèle que la teneur en lipides varie avec la méthode d'extraction.

Les méthodes de SBR, de RöseGottlieb, de Folch et al (1957) et de Clark et al (1982) conduisent à une estimation comparable de la teneur en lipides du lactosérum $(0,7-0,8 \mathrm{~g} / 100 \mathrm{~g}$ de matière sèche). Par contre, la méthode de Wolff et Castera-Rossignol (1987) fournit des valeurs 2 fois plus élevées. Cette valeur par excès peut être attribuée à une contamination de l'extrait lipidique. En effet, après évaporation du mélange isopropanol/ hexane et reprise de l'extrait sec dans le mélange chloroforme/méthanol $(2 / 1)$, un lavage de la phase organique avec une solution aqueuse à $0,73 \%$ de $\mathrm{NaCl}$ pour éliminer les contaminants non lipidiques suivant la procédure décrite dans la méthode de Folch et al (1957) permet d'obtenir une teneur en lipides voisine de celles estimées par les autres méthodes.

Pour le concentré lipoprotéique, les 2 méthodes de référence ne donnent pas des résultats comparables. La teneur en lipides mesurée par la méthode de SBR est supérieure de $0,5 \mathrm{~g}$ pour $100 \mathrm{~g}$ de matière sèche à celle obtenue par la méthode de Röse-Gottlieb. Toutefois, les résultats de ces 2 méthodes ne sont pas significative- 
ment différents de ceux fournis par la méthode de Clark et al (1982). Comme pour le lactosérum, la méthode de Wolff et Castera-Rossignol (1987) conduit à une estimation par excès de la teneur en lipides du concentré lipoprotéique. Un lavage de l'extrait lipidique par une solution saline de $\mathrm{NaCl}$ permet de pallier cet inconvénient. A l'inverse, la méthode de Folch et al (1957) fournit des valeurs par défaut. Ce résultat s'explique sans doute par le fait que l'extraction est pratiquée sur le lyophilisat et non sur l'échantillon réhydraté.

Le traitement préalable du concentré lipoprotéique avec de l'EDTA ou de l'acide effectué pour faciliter la rupture des liaisons lipides-protéines n'améliore pas le rendement de l'extraction comparativement à celui obtenu avec la méthode de Clark et al (1982) pratiquée directement sur le concentré réhydraté.

\section{Proportions relatives des lipides neutres et polaires (tableau II)}

Que ce soit dans le lactosérum ou le concentré lipoprotéique, les proportions relatives de lipides neutres et de phospholipides dans l'extrait lipidique total sont comparables, même dans le cas de la méthode de Folch et al (1957) qui ne conduit qu'à une extraction partielle des lipides du concentré lipoprotéique.

Seule, la méthode de SBR conduit à un rapport lipides neutres/phospholipides beaucoup plus élevé que les autres méthodes. Les extraits lipidiques issus d'un cette technique se composent d'une très forte proportion de lipides neutres et d'un faible taux de phospholipides. Ce résultat peut être attribué à une dégradation partielle des phospholipides par l'acide chlorhydrique (voir paragraphe suivant).

\section{Répartition en classes des phospholipides (tableau III)}

Les répartitions en classes des phospholipides des extraits lipidiques obtenus par les méthodes de Röse-Gottlieb, Folch et al (1957), Clark et al (1982) et Wolff et Castera-Rossignol (1987) sont très voisines. Quelle que soit la méthode considérée, le lactosérum contient environ $30 \%$ de Phosphatidyl-Ethanolamine (PE), $27 \%$ de Phosphatidyl-Inositol + Phosphatidyl-Sérine (dont moins de $1 / 5$ de PS), $25 \%$ de Phosphatidyl-Choline (PC) et 13-16\% de Sphingomyéline. Comparativement, le concentré lipoprotéique est plus pauvre en PI + PS (12 à $18 \%$ ) et nettement plus riche en PC (34 à $42 \%$ ).

Les résultats obtenus avec la méthode SBR n'ont pas été inclus dans le tableau III. En effet, le chromatogramme de l'extrait lipidique total montre que les phospholipides ont pratiquement disparu. De plus, les pics situés dans la zone habituelle d'élution de ces lipides sont de forme très dissymétrique et ne se superposent pas avec les pics caractéristiques des principales classes de phospholipides. Ces composés correspondent sans doute à des produits d'oxydation des phospholipides natifs. Par ailleurs, la surface du pic d'acides gras libres est fortement accrue indiquant une hydrolyse importante des phospholipides sous l'action de l'acide chlorhydrique.

\section{Composition en acides gras (tableaux IV, V, VI)}

\section{Composition en acides gras des lipides totaux (tableau IV)}

La composition en acides gras des lipides totaux du lactosérum et du concentré lipo- 
Tableau II. Influence de la méthode d'extraction sur les teneurs en lipides totaux, lipides neutres (LN) et phospholipides ( $P L$ ) du lactosérum et du concentré lipoprotéique (en g/100 de matière sèche). Effect of the extraction method on total lipid, neutral lipid and phospholipid content of the whey and high lipid content by-product (in g/100 g dry weight).

\begin{tabular}{|c|c|c|c|c|c|c|c|}
\hline Méthodes & $S B R^{1}$ & $R G^{2}$ & Folch $^{3}$ & Clark $^{4}$ & $\begin{array}{c}\text { Clark }^{4} \\
+ \\
\text { EDTA }\end{array}$ & $\begin{array}{c}\text { Clark }^{4} \\
+ \\
\text { acide }\end{array}$ & Wolff \\
\hline \multicolumn{8}{|l|}{ Lactosérum } \\
\hline Lipides totaux & $0,8^{\mathrm{a}}$ & $0,8^{a}$ & $0,7^{a}$ & $0,7^{a}$ & - & - & $\begin{array}{l}1,6^{\mathrm{b}} \\
0,8^{\mathrm{ab}}\end{array}$ \\
\hline Lipides neutres & $0,6^{\mathrm{b}}$ & $0,4^{\mathrm{a}}$ & $0,4^{a}$ & $0,4^{\mathrm{a}}$ & - & - & $0,4^{a}$ \\
\hline Phospholipides & $0,2^{a}$ & $0,3^{a}$ & $0,3^{a}$ & $0,3^{a}$ & - & - & $0,3^{a}$ \\
\hline LN/PL & $2,7^{b}$ & $1,4^{a}$ & $1,6^{a}$ & $1,2^{\mathrm{a}}$ & - & - & $1,2^{a}$ \\
\hline \multicolumn{8}{|c|}{ Concentré lipoprotéique } \\
\hline Lipides totaux & $3,0^{d}$ & $2,5^{c}$ & $1,5^{a}$ & $2,9^{c d}$ & $2,8^{\mathrm{cd}}$ & $2,1^{b}$ & $\begin{array}{l}3,3^{\ominus} \\
2,8^{\text {cd* }}\end{array}$ \\
\hline Lipides neutres & $2,9^{f}$ & $1,5^{c}$ & $0,9^{a}$ & $1,8^{d}$ & $1,8^{d}$ & $1,2^{b}$ & $1,7^{d}$ \\
\hline Phospholipides & $0,1^{\mathrm{a}}$ & 1,0 cd & $0,6^{b}$ & $1,1^{d}$ & $1,0^{\mathrm{cd}}$ & $0,9^{c}$ & $1,1 d$ \\
\hline LN/PL & $24,9^{b}$ & $1,5^{a}$ & $1,5^{a}$ & $1,5^{a}$ & $1,8^{a}$ & $1,3^{a}$ & $1,5^{a}$ \\
\hline
\end{tabular}

1 = méthode de Schmidt-Bondzinski-Ratzlaff (Norme V04-215). 2 = méthode de Röse-Gottlieb (Norme V04-214). 3 $=$ méthode de Folch et al (1957). 4 = méthode de Clark et al (1982) sans traitement préalable de l'échantillon (Clark), avec traitement à l'EDTA (EDTA) ou à l'acide (acide). 5 = méthode de Wolf et Castera-Rossignol (1987). " : valeur obtenue après purification de l'extrait lipidique selon la méthode de Folch et al (1957). Chaque valeur est la moyenne de 4 déterminations. Sur une même ligne, les valeurs surmontées d'une même lettre ne sont pas significativement différentes au seuil de $5 \%$.

1 = Method of Schmidt-Bondzinski-Ratzlaff (Norm V04-215); 2 = method of Röse-Gottlieb (Norm V04-214); $3=$ method of Folch et al (1957); 4 = method of Clark et al (1982) without preliminary treatment of the sample (Clark), with EDTA (EDTA) or acid (acide) addition to the sample; 5 = method of Wolff and Castera-Rossignol (1987). "Value obtained after purification of the lipid extract according to the method of Folch et al (1957). Each number is the mean of 4 determinations. In the same row values followed by the same letter in superscript are not significantly different at the $5 \%$ level.

protéique se caractérise par une forte teneur en acides gras saturés et un faible taux d'acides gras polyinsaturés (63-70\% et $3-5 \%$ respectivement).

Les méthodes d'extraction influent peu sur la composition en acides gras des lipides totaux du lactosérum. Par contre, la composition en acides gras du concentré lipoprotéique varie suivant la méthode considérée. Les taux d'acides gras polyinsaturés sont les plus élevés dans les extraits lipidiques obtenus par la méthode de Clark et al (1982) (5\% des acides gras to- taux) alors que les extraits lipidiques issus de la méthode de SBR en sont très pauvres $(2 \%)$, les autres méthodes d'extraction donnant des résultats intermédiaires $(3,6-3,9 \%)$.

\section{Composition en acides gras des lipides neutres et phospholipides (tableaux V et VI)}

La composition en acides gras des lipides neutres est peu influencée par la méthode 
Tableau III. Influence de la méthode d'extraction sur la répartition en classes des phospholipides du lactosérum et du concentré lipoprotéique (en pourcentage des phospholipides totaux).

Effect of the extraction method on the phospholipid composition of the whey and high lipid content byproduct (in \% of total phospholipids).

\begin{tabular}{|c|c|c|c|c|}
\hline Méthodes & $R G^{2}$ & Folch ${ }^{3}$ & Clark $^{4}$ & Wolff5 \\
\hline \multicolumn{5}{|l|}{ Lactosérum } \\
\hline PE & 32,8 & 30,9 & 32,5 & 32,6 \\
\hline $\mathrm{PI}+\mathrm{PS}$ & 27,3 & 27,0 & 26,5 & 27,9 \\
\hline PC & 25,7 & 25,4 & 27,5 & 24,3 \\
\hline $\mathrm{SPH}$ & 14,2 & 16,7 & 13,1 & 14,2 \\
\hline PE/PC & 1,3 & 1,2 & 1,2 & 1,3 \\
\hline \multicolumn{5}{|c|}{ Concentré lipoprotéique } \\
\hline PE & 27,1 & 28,2 & 30,5 & 29,5 \\
\hline$P I=P S$ & 12,9 & 18,6 & 16,0 & 15,0 \\
\hline $\mathrm{PC}$ & 42,2 & 33,7 & 36,9 & 36,4 \\
\hline $\mathrm{SPH}$ & 16,8 & 19,2 & 22,2 & 18,8 \\
\hline $\mathrm{PE} / \mathrm{PC}$ & 0,7 & 0,8 & 0,8 & 0,8 \\
\hline
\end{tabular}

Pour les abréviations des méthodes d'extraction se reporter au tableau II.

See table II for extraction method abbreviations.

$\mathrm{PE}=$ Phosphatidyl-ethanolamine $\mathrm{PI}+\mathrm{PS}=$ phosphatidyl-inositol et phosphatidyl-sérine; $\mathrm{PC}=$ phosphadityl-choline, $\mathrm{SPH}=$ sphingomyéline.

d'extraction. En effet, seuls les lipides neutres de l'extrait lipidique obtenu par la méthode de SBR se distinguent de ceux obtenus par les autres méthodes par une teneur en acides gras polyinsaturés nettement plus importante $(5,5 \%$ contre $3,2 \%$ dans le lactosérum, 4,2\% contre 2,2 à $3,0 \%$ dans le concentré lipoprotéique).

Le taux d'acides gras polyinsaturés est plus élevé dans les phospholipides du lactosérum que dans ceux du concentré lipoprotéique $(6,9-10,5 \%$ au lieu de 5,7$7,1 \%$ ). A l'inverse, cette fraction est plus riche en acides gras mono-insaturés dans le lactosérum que dans le concentré.

La méthode d'extraction affecte significativement le taux d'acides gras polyinsaturés des phospholipides. Aussi bien dans le lactosérum que dans le concentré, les phospholipides sont plus riches en acides gras polyinsaturés quand l'extrait lipidique est obtenu par la méthode de Clark et al (1982) que lorsqu'il est obtenu par les méthodes de Wolff et Castera-Rossignol (1987) et de Folch et al (1957) (tableau VI). Les phospholipides du concentré lipoprotéique extraits par la méthode de RöseGottlieb présentent un taux d'acides gras polyinsaturés voisin de ceux extraits par les méthodes de Wolff et CasteraRossignol (1987) et de Folch et al (1957). Par contre, dans le lactosérum, les phospholipides extraits par la méthode de Röse-Gottlieb contiennent significativement moins d'acides gras polyinsaturés (tableau VI). 
Tableau IV. Influence de la méthode d'extraction sur la composition en acides gras des lipides totaux du lactosérum et du concentré lipoprotéique (en pourcentage de la surface des esters méthyliques). Effect of the extraction method on the fatty acid composition of total lipids of the whey and the high lipid content by-product (in \% of the area of methylesters).

\begin{tabular}{|c|c|c|c|c|c|c|c|}
\hline Méthodes & $S R B^{1}$ & $R G^{2}$ & Folch $^{3}$ & Clark ${ }^{4}$ & $\begin{array}{c}\text { Clark }^{4} \\
+ \\
\text { EDTA }\end{array}$ & $\begin{array}{c}\text { Clark }^{4} \\
+ \\
\text { acide }\end{array}$ & Wolffe \\
\hline \multicolumn{8}{|l|}{ Lactosérum } \\
\hline Saturés (S) & $63,8^{a}$ & 64,8 ab & $66,0^{\mathrm{b}}$ & $64,9^{a b}$ & & & $65,6^{a b}$ \\
\hline Mono-insaturés & $30,7^{a}$ & $30,2^{a}$ & $29,3^{a}$ & $29,8^{a}$ & & & $29,5^{a}$ \\
\hline Polyinsaturés $(P)$ & $5,5^{\mathrm{b}}$ & $5,0^{a b}$ & $4,7^{a}$ & $5,2^{a b}$ & & & $4,9 a b$ \\
\hline $\mathrm{P} / \mathrm{S}$ & $0,09^{a}$ & $0,08^{a}$ & $0,07^{a}$ & $0,08^{a}$ & & & $0,07^{a}$ \\
\hline \multicolumn{8}{|c|}{ Concentré lipoprotéique } \\
\hline Saturés (S) & $70,9^{\mathrm{c}}$ & $65,7^{b}$ & $66,0^{\mathrm{b}}$ & $63,2^{a}$ & $63,4^{a}$ & $63,6^{a}$ & $64,5^{a b}$ \\
\hline Mono-insaturés & $26,8^{a}$ & $30,7 \mathrm{bc}$ & $30,0^{\mathrm{b}}$ & $31,8^{c}$ & $31,9^{\mathrm{c}}$ & $31,9 c$ & $31,7^{\mathrm{c}}$ \\
\hline Polyinsaturés $(P)$ & $2,0^{a}$ & $3,6^{b}$ & $3,9 \mathrm{bc}$ & $5,0^{d}$ & $4,6^{\mathrm{cd}}$ & $4,4^{b c d}$ & $3,8^{b c}$ \\
\hline P/S & $0,03^{a}$ & $0,06^{b}$ & $0,06^{b c}$ & $0,08^{d}$ & 0,07 cd & $0,06^{c d}$ & $0,06^{b c}$ \\
\hline
\end{tabular}

Pour les abréviations des méthodes d'extraction se reporter au tableau II. Chaque valeur est la moyenne de 4 déterminations. Sur une même ligne, les valeurs surmontées d'une même lettre ne sont pas significativement différentes au seuil de $5 \%$.

See table II for extraction abbreviations. Each number is the mean of 4 determinations. In the same row, values followed by the same letter in superscript are not significantly different at the $5 \%$ level.

\section{DISCUSSION}

\section{Teneur en lipides}

Trois méthodes (SBR (1963), Röse Gottlieb (1962), Clark et al (1982) donnent des estimations comparables de la teneur en lipides du lactosérum et du concentré lipoprotéique. Soulignons que la méthode de SBR tend à surestimer légèrement le taux de lipides des 2 produits étudiés. $\mathrm{Ce}$ fait a été souligné par d'autres auteurs lors de l'extraction des lipides du lait. Ils expliquent ces valeurs par excès par la présence dans l'extrait lipidique de contaminants non lipidiques, en particulier de produits de dégradation du lactose (Walstra et Mulder, 1963).
La méthode de Folch et al (1957) donne des résultats comparables à ceux des 3 méthodes citées ci-dessus pour le lactosérum. Par contre, elle n'extrait qu'une faible proportion $(50 \%)$ des lipides du concentré lipoprotéique. Ce résultat peut être expliqué par l'incapacité du mélange solvant chloroforme/méthanol, en l'absence d'eau, à rompre les liaisons lipides-protéines formées lors de l'agrégation thermocalcique des lipides suivant le procédé décrit par Fauquant et al (1985a).

La méthode de Wolff et CasteraRossignol (1987) conduit à une surestimation très nette du taux de lipides du lactosérum et du concentré lipoprotéique. Ce résultat est attribué à la présence de contaminants dans l'extrait lipidique. Nous avons pu le montrer en solubilisant les li- 
Tableau V. Influence de la méthode d'extraction sur la composition en acides gras des lipides neutres du lactosérum et du concentré lipoprotéique (en pourcentage de la surface des esters méthyliques).

Effects of the extraction method on fatty acid composition of neutral lipids of the whey and high lipid content by-product (in \% of the area of methylesters).

\begin{tabular}{|c|c|c|c|c|c|c|c|}
\hline Méthodes & $S B R^{1}$ & $R G^{2}$ & Folch ${ }^{3}$ & Clark $^{4}$ & $\begin{array}{c}\text { Clark }^{4} \\
+ \\
\text { EDTA }\end{array}$ & $\begin{array}{c}\text { Clark }^{4} \\
+ \\
\text { acide }\end{array}$ & Wolff ${ }^{5}$ \\
\hline \multicolumn{8}{|l|}{ Lactosérum } \\
\hline Saturés (S) & $63,8^{b}$ & $71,0^{a}$ & $72,3^{a}$ & $71,7^{a}$ & - & - & $73,0^{\mathrm{a}}$ \\
\hline Mono-insaturés & $30,7^{b}$ & $25,4^{a}$ & $24,3^{a}$ & $25,0^{a}$ & - & - & $23,7^{a}$ \\
\hline Polyinsaturés $(P)$ & $5,5^{b}$ & $3,6^{a}$ & $3,3^{a}$ & $3,2^{a}$ & - & - & $3,2^{a}$ \\
\hline $\mathrm{P} / \mathrm{S}$ & $0,09^{b}$ & $0,05^{a}$ & $0,05^{a}$ & $0,05^{a}$ & - & - & $0,05^{a}$ \\
\hline \multicolumn{8}{|c|}{ Concentré lipoprotéine } \\
\hline Saturés (S) & $65,8^{a}$ & $74,3^{\mathrm{b}}$ & $73,4^{b}$ & $71,7^{b}$ & $70,5^{b}$ & $71,9^{b}$ & $72,4^{b}$ \\
\hline Mono-insaturés & $29,9^{c}$ & $23,5^{a}$ & $24,2^{\mathrm{ab}}$ & $25,6^{a b}$ & $26,5^{b}$ & $25,8^{a b}$ & $25,3^{a b}$ \\
\hline Polyinsaturés $(P)$ & $4,2^{b}$ & $2,2^{\mathrm{a}}$ & $2,3^{a}$ & $2,7^{a}$ & $3,0^{a}$ & $2,3^{a}$ & $2,3^{a}$ \\
\hline P/S & $0,07^{b}$ & $0,02^{a}$ & $0,03^{a}$ & $0,04^{a}$ & $0,04^{a}$ & $0,04^{a}$ & $0,03^{a}$ \\
\hline
\end{tabular}

Pour les abréviations des méthodes d'extraction se reporter au tableau II. Chaque valeur est la moyenne de 4 déterminations. Sur une même ligne, les valeurs surmontées d'une même lettre ne sont pas significativement différentes au seuil de $5 \%$.

See table II for extraction method abbreviations. Each number is the mean of 4 determinations. In the same row, values followed by the same letter in superscript are not significantly different at the $5 \%$ levell.

pides dans le mélange chloroforme/ méthanol puis en lavant la phase organique avec une solution aqueuse à $0,73 \%$ de $\mathrm{NaCl}$ comme cela est pratiqué dans les méthodes de Clark et al (1982) et Folch et al (1957). En effet, cette procédure permet de rapprocher les estimations des taux de lipides obtenus par la méthode de Wolff et Castera-Rossignol (1987) de celles mesurées par la méthode de SBP. de Röse Gottlieb et de Clark et al (1982). Ce résultat atteste que la simple filtration sur célite de l'échantillon en suspension dans l'isopropanol-hexane est insuffisante pour retenir les contaminants non lipidiques (protéines, lactose). Cette constatation avait déjà été faite par Marmer et Maxwell (1981) qui préconisaient d'ajouter un lit composé de célite et de calcium hydrogénophosphate dans la partie inférieure de la colonne de filtration pour retenir les protéines entraînées par le solvant. Toutefois, cette précaution se révèle insuffisante pour éviter toute contamination des lipides. En effet, les teneurs en lipides de divers produits laitiers déterminées suivant la méthode de Maxwell et al (1986) restent significativement plus élevées que celles mesurées par la méthode de RöseGottlieb (Maxwell et al, 1986). Par ailleurs, nous avons pu établir que le lactose est partiellement entraîné au travers de la colonne de célite par le mélange solvant hexane/isopropanol. Cette contamination de l'extrait lipidique par le lactose pourrait être la cause principale des valeurs excessives observées avec la méthode de Wolff et Castera-Rossignol (1987) puisque le lactosérum et le concentré lipoprotéique sont riches en lactose. 
Tableau VI. Influence de la méthode d'extraction sur la composition en acides gras des phospholipides du lactosérum et du concentré lipoprotéique (en pourcentage de la surface des esters méthyliques).

Effect of the extraction method on fatty acid composition of phospholipids of whey and high lipid content by-product (in \% of the area of methylesters).

SBR $^{1} \quad \mathrm{RG}^{2} \quad$ Folch $^{3} \quad$ Clark $^{4} \underset{\text { EDTA }}{\text { Clark }^{4}} \begin{aligned} & \text { Clark }^{4}+ \\ & \text { acide }\end{aligned}$ Wolff $^{5}$

\begin{tabular}{|c|c|c|c|c|c|c|c|}
\hline \multicolumn{8}{|l|}{ Lactosérum } \\
\hline Saturés (S) & - & $56,9^{b}$ & $44,7^{\mathrm{a}}$ & $48,3^{a}$ & - & - & $49,2^{\mathrm{a}}$ \\
\hline Mono-insaturés & - & $36,2^{\mathrm{a}}$ & $45,9^{b}$ & $41,1^{\mathrm{ab}}$ & - & - & $41,5^{\mathrm{ab}}$ \\
\hline Polyinsaturés $(P)$ & - & $6,9^{a}$ & $9,3^{\mathrm{b}}$ & $10,5^{b}$ & _- & _- & $9,2^{\mathrm{b}}$ \\
\hline $\mathrm{P} / \mathrm{S}$ & - & $0,12^{a}$ & $0,21^{b}$ & $0,22^{b}$ & - & - & $0,19^{b}$ \\
\hline \multicolumn{8}{|c|}{ Concentré lipoprotéique } \\
\hline Saturés (S) & - & $54,1^{b c}$ & $54,8^{c}$ & $47,9^{a b}$ & $45,3^{a}$ & $49,8^{a b c}$ & $53,4^{\text {bc }}$ \\
\hline Mono-insaturés & - & $40,1^{a}$ & $39,6^{\mathrm{a}}$ & $47,7^{b}$ & $47,7^{b}$ & $43,8^{a b}$ & $40,9^{a}$ \\
\hline Polyinsaturés (P) & - & $5,7^{a b}$ & $5,6^{a}$ & $7,1^{\mathrm{c}}$ & $6,9^{c}$ & $6,4 b^{c}$ & $5,7^{a b}$ \\
\hline $\mathrm{P} / \mathrm{S}$ & - & $0,11^{\mathrm{a}}$ & $0,10^{\mathrm{a}}$ & $0,15^{b}$ & $0,15^{b}$ & $0,13^{a b}$ & $0,11^{\mathrm{a}}$ \\
\hline
\end{tabular}

Pour les abréviations des méthodes d'extraction se reporter au tableau II. Chaque valeur est la moyenne de 4 déterminations. Sur une même ligne, les valeurs surmontées d'une même lettre ne sont pas significativement différentes au seuil de $5 \%$.

See table II for extraction method abbreviations. Each number is the mean of 4 determinations. In the same row, values followed by the same letter in superscript are not significantly different at the $5 \%$ level.

Soulignons que les 2 modifications que nous proposions pour tenter de faciliter l'extraction des lipides du concentré lipoprotéique (ajout d'EDTA ou ajustement du $\mathrm{pH}$ à 4,6), n'ont apporté aucune amélioration du rendement d'extraction. Bien plus, l'acidification de l'échantillon a eu un effet négatif. Ces méthodes qui ajoutent une étape supplémentaire à l'extraction sont donc inefficaces.

\section{Taux et répartition en classes des phospholipides}

Les phospholipides possèdent des propriétés amphiphiles qui en font des molécules très recherchées dans le domaine de la technologie. Toutefois, ce sont les lipides les plus difficiles à extraire car ils sont souvent associés aux protéines ou aux poly- saccharides (Zhukov et Vereshchagin, 1981; Christie, 1987). De plus, ces lipides sont particulièrement sensibles à l'oxydation et à l'hydrolyse. De nombreuses méthodes ont été critiquées parce qu'elles n'assurent pas une extraction totale des phospholipides et/ou provoquent leur altération partielle. C'est pourquoi les mesures du taux de phospholipides et de leur répartition en classes peuvent être considérées comme de bons indices pour évaluer les qualités d'une méthode d'extraction.

La méthode de SBR est à proscrire dès lors qu'une caractérisation des lipides est envisagée. En effet, cette méthode dégrade très fortement les phospholipides. Cette conclusion s'appuie sur plusieurs observations concordantes :

- un rapport lipides neutres/phospholipides très élevé dans les extraits lipidi- 
ques qui dénote un recouvrement incomplet des phospholipides;

- des profils chromatographiques de la fraction phospholipidique atypiques qui montrent une dégradation importante des principaux phospholipides;

- la présence d'une proportion élevée d'acides gras polyinsaturés dans les lipides neutres qui témoigne d'une hydrolyse importante des phospholipides.

Ces observations sont en accord avec celles rapportées par Kuzdzal-Savoie (1971).

Les 4 autres méthodes étudiées conduisent à une estimation comparable de la proportion de phospholipides dans les extraits lipidiques. De plus, la répartition en classes de phospholipides est très proche quelle que soit la méthode considérée (tableau III). II est remarquable de souligner que ce résultat reste vrai pour la méthode Folch et al (1957) qui pourtant n'extrait qu'une partie des lipides totaux du concentré lipoprotéique. II semble donc que ces 4 méthodes permettent une caractérisation de la fraction phospholipidique puisqu'elles ne sont pas sélectives vis-à-vis des principales classes de phospholipides.

\section{Taux d'acides gras polyinsaturés}

L'évaluation du taux d'acides gras polyinsaturés d'un aliment ou d'une matière première revêt un grand intérêt tant sur le plan nutritionnel que technoiugique. En effet, les acides gras polyinsaturés sont pour la plupart des acides gras essentiels dont les rôles biologiques ont été largement étudiés (Holman, 1986). De plus, ces acides gras sont sensibles à l'oxydation, et à ce titre, ils influent très largement sur l'aptitude à la transformation et à la conservation des matières premières et des produits finis. II est donc indispensable que les différentes étapes des méthodes d'extraction n'oxydent pas les acides gras polyinsaturés. Attendu qu'il est pratiquement impossible de prévenir totalement les lipides de l'oxydation, il est généralement admis de considérer la méthode qui permet d'obtenir les extraits lipidiques les plus riches en acides gras polyinsaturés comme celle qui est la moins oxydante visà-vis de ces acides gras.

Les compositions en acides gras des lipides totaux, neutres et polaires du lactosérum et du concentré lipoprotéique sont très proches de celles publiées antérieurement pour les lipides du lait (Jensen, 1973). Les extraits lipidiques totaux et les lipides neutres du lactosérum présentent des taux d'acides gras polyinsaturés très voisins, quelle que soit la méthode d'extraction considérée.

Toutefois, les méthodes d'extraction à froid avec un solvant binaire (Folch et al, (1957), Clark et al (1982), Wolff et Castera-Rossignol (1987)) semblent moins oxyder les acides gras polyinsaturés dans les phospholipides que celle de Röse-Gottlieb. Cette dégradation partielle des acides gras polyinsaturés lors de l'extraction par la méthode de Röse-Gottlieb est sans doute la conséquence du traitement thermique en présence d'ammoniaque qui précède l'extraction des lipides.

Dans le cas du concentré lipoprotéique, il faut souligner que la méthode de Clark et al (1982) fournit les extraits lipidiques totaux et les phospholipides les plus riches en acides gras polyinsaturés. Les méthodes de Wolff et Castera-Rossignol (1987), Folch et al (1957) et Röse-Gottlieb conduisent à des compositions en acides gras des lipides totaux, neutres et polaires très voisines. Les faibles taux d'acides gras polyinsaturés des phospholipides extraits par la méthode de Folch et al (1957) et Wolff et Castera-Rossignol (1987) par rapport à ceux des phospholipides extraits 
par la méthode de Clark demeurent inexpliqués, ces méthodes étant généralement considérées comme peu oxydantes.

\section{CONCLUSION}

Sur la base des 5 critères de comparaison que nous avons retenus, il s'avère que la meilleure méthode serait la méthode de Clark et al (1982). Pour les 2 matières premières étudiées, elle fournit une bonne estimation de la teneur en lipides, elle permet une extraction totale des phospholipides sans provoquer d'oxydation importante des acides gras polyinsaturés. Si l'intérêt de cette méthode est indiscutable dès lors qu'il s'agit de caractériser précisément la fraction lipidique du lactosérum et du concentré lipoprotéique, la durée de l'extraction et la quantité de solvant mise en œuvre interdisent pratiquement son utilisation en routine. Cette méthode semble s'adresser avant tout aux laboratoires de recherche.

Dans un laboratoire de contrôle, la méthode Röse-Gottlieb peut constituer un compromis acceptable. Rapide, elle permet une estimation correcte de la teneur en lipides des produits laitiers. L'extraction des phospholipides peut être considérée comme satisfaisante puisqu'aucune discrimination des principales classes de phospholipides n'a été observée. Toutefois, elle semble altérer légèrement les acides gras polyinsaturés des phospholipides.

La méthode de Wolff et CasteraRossignol (1987) apparaît comme une alternative intéressante. En effet, même si cette méthode conduit à une estimation par excès de la teneur en lipides, elle est aussi rapide que celle de Röse-Gottlieb et fournit des résultats comparables pour la répartition en classes des phospholipides et la composition en acides gras des lipides. De plus, il est toujours possible de procéder à un lavage de la phase organi- que contenant l'extrait lipidique par une solution saline pour éliminer les contaminants, sans que la durée de l'analyse soit considérablement accrue. Par ailleurs, il faut rappeler que l'intérêt essentiel de cette méthode réside dans la possibilité de préparer directement des esters isopropyliques des acides gras qui facilite grandement la détermination de la composition en acides gras des fractions lipidiques (Wolff et Fabien, 1989).

La méthode de Folch et al (1957) semble peu adaptée à l'extraction des lipides du lactosérum et de ses dérivés en raison des risques importants de former des suspensions stables au cours de la procédure. La méthode de Maxwell et al (1986) qui fait appel à un mélange solvant voisin de celui utilisé dans la méthode de Folch et al (1957) mais à une extraction sur colonne sèche comme dans la méthode de Wolff et Castera-Rossignol (1987) permet de pallier cet inconvénient. Toutefois, les essais que nous avons pratiqués avec la méthode de Maxwell et al (1986) se sont heurtés à 2 difficultés :

- soit une contamination excessive des extraits lipidiques par d'autres constituants comme dans la méthode de Wolff et Castera-Rossignol (1987);

- soit un écoulement trop rapide du solvant au travers de la colonne qui conduit à une extraction incomplète des lipides.

La méthode de SBR semble à proscrire pour 2 raisons essentielles:

- elle tend à surestimer la teneur en lipides des concentrés lipoprotéiques;

- elle altère les lipides interdisant toute détermination qualitative ultérieure.

\section{REMERCIEMENTS}

Les auteurs remercient vivement les chercheurs du laboratoire de technologie laitière de I'INRA 
de Rennes qui ont été à l'origine de ces travaux et la Coopérative laitière de la région nantaise (Colarena, Herbignac) qui nous a fourni gracieusement les échantillons de lactosérum et de concentré lipoprotéique

\section{RÉFÉRENCES}

AFNOR (1986) Recueil de normes françaises. Contrôle de la qualité des produits laitiers. AFNOR, Paris-la-Défense

Bartiett G (1959) Phosphorus assay in column chromatography. J Biol Chem 234, 466-468

Castera A, Mordret F, Chazan JB (1989) Evaluation et généralisation d'une méthode d'extraction de la matière grasse des produits alimentaires. In: Actes du Congrès International Chevreul pour l"Étude des Corps gras T1, ETIG, Paris, 272-276

Cerbulis $\mathrm{J}$, Woychik $\mathrm{JH}$, Wondolowski MV (1972) Composition of commercial wheys. J Agric Food Chem 20, 1057-1059

Christie WW (1987) Prelude to analysis: Extraction, storage and preliminary fractionation of lipids. In: HPLC and Lipids, a Practical Guide (Christie WW, ed) Pergamon Press, Oxford, 71-86

Clark RM, Ferris AM, Fey M, Brown PB, Hundrieser KE, Jensen RG (1982) Changes in the lipids of human milk from 2 to 16 weeks postpartum. I Pediatr Gastroenterol Nutr 1, 311-315

Fauquant J, Pierre A, Brulé G (1985a) Clarification du lactosérum acide de caséinerie. Tech Lait 1003, 37-41

Fauquant J, Vieco E, Brulé G, Maubois JL (1985b) Clarification des lactosérums doux par agrégation thermocalcique de la matière grasse résiduelle. Lait 65, 647-648

Folch J, Lees M, Sloane-Stanley GA (1957) A simple method for isolation and purification of total lipids from animal tissues. J Biol Chem 266, 497-509

Hara A, Radin N (1978) Lipid extraction of tissues with a low toxicity solvent. Anal Biochem $90,420-426$

Holman RT (1986) Essential fatty acids, prostaglandins and leucotrienes. Progr Lipid Res $25,693 p$
Jensen RG (1973) Composition of bovine milk lipids. J Am Oil Chem Soc 50, 186-192

Juaneda P, Rocquelin G (1985) Rapid and convenient separation of phospholipids and no phosphorus lipids from rat heart using silica cartridges. Lipids 20, 40-41

Kuzdzal-Savoie S (1971) Obtention de matière grasse du lait (liquide ou en poudre). Tech Lait 690, 9-12

Leseigneur A, Gandemer G, Marion D (1989) Fractionnement en classes des lipides alimentaires par HPLC à l'aide d'un détecteur à diffusion de lumière. In: Actes du Congrès Internationl Chevreul pour l'Étude des Corps Gras, T1. Etig, 311-318

Marmer WN, Maxwell RJ (1981), Dry column method for quantitative extraction and simultaneous class separation of lipids from muscle tissue. Lipids 16, 365-371

Maubois JL, Pierre A, Fauquant J, Piot M (1987) Industrial fractionation of main whey proteins. Bull IDF 212, 154-159

Maxwell RJ, Mondimore D, Tobias J (1986) Rapid method for the quantitative extraction and simultaneous class separation of milk lipids. J Dairy Sci 69, 321-325

Morrison WR, Smith LM (1964) Preparation of fatty acid methyl esters and dimethylacetals from lipids with boron-fluoride methanol. $J$ Lipid Res 5, 600-608

Timmen H, Dimick PS (1972) Structure and synthesis of milk fat. $X$. characterisation of the major hydroxy compounds in milk lipids. $J$ Dairy Sci 55, 919-925

Walstra P, Mulder H (1963) Gravimetric methods for the determination of the fat content of milk and milk products. III. The SchmidBondzynski-Ratzlaff method. Neth Milk Dairy J17, 334-346

Wolff RL, Castera-Rossignol AFM (1987) Mise au point d'une méthode d'extraction de la matière grasse de fromage de type emmental. Rev Fr Corps Gras 34, 123-132

Wolff RL, Fabien RJ (1989) Utilisation de l'isopropanol pour l'extraction de la matière grasse de produits laitiers et pour l'estérification subséquente des acides gras. Lait 69, 33-46

Zhukov AV, Vereshchagin AG (1981) Current techniques of extraction, purification and preliminary fractionation of polar lipids of natural origin. Adv Lipid Res 18, 247-282 